\title{
Prospects for Stratified and Precision Medicine in Systemic Sclerosis Treatment
}

\author{
Kristina E. N. Clark, BA, MBBS, MRCP ${ }^{1}$ \\ Emma Derrett-Smith, PhD, MRCP ${ }^{1,2, *}$
}

\author{
Address \\ ${ }^{*}$ CCentre for Rheumatology and Connective Tissue Diseases, University College \\ London Division of Medicine, London, U.K. \\ Email: e.derrett-smith@ucl.ac.uk \\ ${ }^{2}$ University Hospitals Birmingham NHS Foundation Trust, Birmingham, U.K.
}

Published online: 20 May 2019

(C) The Author(s) 2019

This article is part of the Topical Collection on Scleroderma

Keywords Systemic sclerosis · Scleroderma $\cdot$ Stratification $\cdot$ Biomarkers $\cdot$ Gene expression

\begin{abstract}
Purpose of review Precision medicine is an evolving field stemming from Oncology research, with an increasingly important role in autoimmune diseases. The heterogeneity, both of clinical presentations of systemic sclerosis and differing response to treatment, emphasises the importance of developing means of patient stratification to ensure that the correct patients are managed with the most appropriate treatments at a disease duration when this will have meaningful impact on disease course and resolution. This review aims to discuss the different means explored so far in stratifying patients with systemic sclerosis. We highlight recent clinical trials which have applied stratification techniques in order to provide a form of precision medicine in the management of systemic sclerosis.

Recent findings Advances have focused on utilising gene expression techniques on whole skin biopsies or fibroblasts to understand which groups of patients are more likely to respond to which treatments. This technique has been used successfully to understand the effect of tocilizumab, abatacept, and fresolimumab on systemic sclerosis, and helped identify those that are more likely to respond to treatment.

Summary Utilising high output platforms to stratify patients for targeted treatment is still in its infancy but has huge potential for ensuring the patients most likely to respond to a specific therapy are put forward to trials. It has already been shown to be successful in those with a high IL-6 profile and will most likely prove hugely informative in the future.
\end{abstract}




\section{Introduction}

Precision medicine is an evolving approach to patientcentred care. Pioneered by cancer medicine, it aims to integrate individual genomics, proteomics, pharmacogenomics, and immunogenomics information to tailor personalised therapy, optimise efficacy, and minimise drug toxicity [1]. By identifying key patient groups, this allows for individualised targeted therapies with increased efficacy and/or reduced toxicity.

Systemic sclerosis (scleroderma; SSc) is a heterogeneous disease characterised by autoimmunity, vasculopathy and fibrosis. Traditionally considered a disease primarily of the skin, the significant internal organ manifestations including lung fibrosis, pulmonary arterial hypertension, gastrointestinal disease, and myositis are responsible for the high case-specific mortality and morbidity in this condition. Disease stratification within SSc has been primarily by extent of skin involvement (limited vs diffuse), by autoantibodies [2] or by organ manifestation. More modern approaches focus on the individual disease process, which targets treatment to either immune activation, vascular disease, or fibrosis, thus aiding with potential targeted therapies.

Biomarkers are a means to aid stratification. The NIH define a biomarker as "a characteristic that is objectively measured and evaluated as an indicator of normal biological processes, pathogenic processes, or pharmacologic responses to a therapeutic intervention" [3]. So far, there is no validated molecular biomarker that meets these criteria in SSc, but there are molecular markers and imaging which correlates with different aspects and stages of the disease.

Lessons learned from the use of targeted treatments in rheumatology and other immunological diseases, combined with the heterogeneity of SSc, suggest that only certain subgroups of patients will benefit from this approach. Emphasis on advancing stratification and precision medicine has never been greater. Given the diverse clinical characteristics of this disease, many clinical trials fail: They are either underpowered or have failed due to the challenges of interpreting any clinical changes. Ensuring the right patients who are likely to benefit from a therapy are entered into the appropriate clinical trial is vital if we are to prove efficacy of specific treatment in SSc. This may be disease subset, stage, or organ manifestation specific, and as advancements in molecular identification continue, this will also include specific biomarkers and genomics hopefully to allow us to fine tune these findings further.
In this article, we look at the different means of stratifying SSc, and how this can be used for personalised therapy. We will cover stratification by organ manifestation, individual biomarkers, and then the natural evolution to microarray and groups of biomarkers.

\section{Stratification by organ manifestation}

\section{Active skin disease}

The degree of skin involvement has historically been the main clinical tool used to stratify patients in SSc, with limited systemic sclerosis (lcSSc) patients having skin involvement limited to below the elbow and knee, and diffuse SSc (dcSSc) being defined as above the elbow and knee including chest. The modified Rodnan skin score (mRSS) is the most frequently used outcome measure, providing a semi-quantitative evaluation of skin thickness [4]. Rapid increase in mRSS in patients with $\mathrm{dcSSc}$ is often associated with new or worsening internal organ involvement, and correlates with increased mortality [5]. The considerable inter-observer variability means that skin score is not a reliable enough predictor of disease severity, trajectory, or response to enable useful stratification alone.

The European Scleroderma Observational Study aimed to derive a prediction model for skin progression in early dcSSc based on clinical characteristics. They identified progressors (defined as an increase in $>5$ units and 25\% increase in mRSS over 12 months) as having a shorter disease duration at time of recruitment, and lower mRSS (although over $45 \%$ had an mRSS of > 22). The interaction with autoantibody status also played a significant role with anti-RNA polymerase III patients having the highest peak skin score, and peaked earlier compared to other autoantibody subtypes [6]. Incorporating initial skin score, with disease duration and autoantibodies improved the accuracy of the model in identifying high-risk patients for skin progression.

\section{Interstitial lung disease}

Clinically significant interstitial lung disease (ILD) affects up to $60 \%$ of patients with SSc [7]. Diagnosis relies on lung function tests and high-resolution chest tomography (HRCT). Those with anti-topoisomerase I 
antibodies are at higher risk, with the presence of centromere antibodies considered protective [7]. Earlier diagnosis would allow earlier initiation of therapy thus halting progression and symptomatic disease.

The recent SPAR model aimed to identify clinical predictors of ILD progression in patients with mild ILD at baseline $(<20 \%$ on HRCT). Validated on two independent cohorts, lower SpO2 after the 6-min walk test (6MWT) and arthritis were identified as independent predictors for ILD progression [8]. Utilising these two parameters, a SPAR score of 2 predicted a rate of ILD progression over $85 \%$, whereas a SPAR score of 0 was $7.4 \%$. However, the validation cohorts did not include anyone with concomitant pulmonary hypertension, and follow-up was only 1 year.

Looking at an unselected SSc cohort over a 15 -year period, variables predictive of clinically significant ILD were greater age at disease onset, dcSSc, lower FVC and lower diffusing capacity for carbon monoxide, and the presence of anti-topoisomerase I antibody [7].

Three promising molecular markers in SSc-ILD highlighted in a recent study include SP-D (surfactant protein), KL-6 (Krebs von den Lungen-6), and CCL19. SP-D was the superior biomarker for diagnosis, KL-6 levels correlated with severity of SSc-ILD, while CCL19 was a predictive biomarker of SSc-ILD progression [9]. Other molecular markers are mentioned later in the text, but those under current investigation include mucin-1 (MUC-1), and CA15-3 which seem to be reliable predictors for cases of more than $20 \%$ fibrosis, where specific immunosuppressive therapy is considered to be indicated [10].

The PROFILE study into idiopathic pulmonary fibrosis (IPF) identified four serum biomarkers predictive of disease progression. These were SP-D, matrix metalloproteinase 7, CA19-9, and CA-125 [11]. Baseline SP-D was significantly higher in patients with progressive disease, and rising concentrations of CA-125 were associated with increased mortality. A rise in collagen degradation markers was strongly predictive of increased mortality, with the extent of change being associated with speed of deterioration [12]. Whether these findings are reproducible to help predict progression in SSc-ILD remains to be clarified.

\section{Pulmonary arterial hypertension}

PAH now carries the highest mortality for patients with SSc [13]. Survival rates are improving, with the recent PHAROS registry reporting 93\%, 75\%, and 49\% survival at 1,3 , and 8 years, respectively. Significant risk factors for poor survival were low DLCO, pericardial effusion, decreased 6-min walk test, low cardiac index, increased mean pulmonary artery pressure, and mean right atrial pressure. Increased age and male gender were poor prognostic signs.

A prediction model identified greater age at onset, increase in serum creatinine levels, lower DLCO, and the presence of anti-RNA polymerase III or anti-U3 RNP antibodies as associated with increased risk of pulmonary hypertension [7]. The presence of antitopoisomerase I antibody reduced the risk of PAH.

NT-proBNP is the most studied biomarker in SSc$\mathrm{PAH}$ and has been demonstrated as a prognostic predictor at baseline in PAH [14]. Changes in NT-proBNP during follow-up aid risk stratification of patients with SSc-PAH. However, raised concentrations of NT-proBNP are not specific to SSc-PAH. Given that RV strain occurs relatively late in $\mathrm{PAH}$, it would be desirable to identify a biomarker which is specific to SSc-PAH, and correlates with early changes in PA pressure.

The DETECT score was the first evidence-based algorithm for detection of patients at risk of PAH [15] and aided in identifying the patients who may need a right heart catheter to determine diagnosis. It utilises lung function, ECG and echocardiography parameters, serum urate and NTproBNP. It has high sensitivity, but a lower specificity, thus avoiding missed diagnoses of PAH. There is no validated tool which exists to identify progression risk or treatment response, although several molecular marker assessments and prediction tools have been recently published.

VEGF-A is a regulator of angiogenesis, and concentrations are increased in patients with PAH [16]. In relatively small studies, VEGF-A was increased in SSc-PAH compared to both healthy controls, and those with SSc without PAH. Levels correlated with systolic pulmonary arterial pressure, DLCO, and MRC dyspnoea score. Further work is required to understand whether VEGF-A has utility as a predictive marker of PAH.

Few biomarkers have been definitive predictors of PAH disease progression. Growth Differentiation Factor-15 (GDF-15) clearly differentiates between SSc$\mathrm{PAH}$ and SSc without PAH, as well as correlating with echocardiographic RVSP but not invasive hemodynamics. Its evidence in idiopathic PAH is stronger for predicting survival [17]. Thrombomodulin is another biomarker found in higher concentrations in SSc-PAH compared to SSc with no PAH and healthy controls [18]. 


\section{Stratification by autoantibodies}

Current stratification methods in SSc rely on extent of skin involvement and autoantibody status. Certain autoantibodies are associated with increased risk of internal organ involvement (Table 1). Anti-topoisomerase (or Scl70) has a strong association with pulmonary fibrosis, whereas anti-RNA-polymerase III is strongly associated with dcSSc (up to 93\% with this antibody have dcSSc), and up to $43 \%$ of patients developing scleroderma renal crisis (SRC). Anti-centromere antibodies (ACA) are the commonest antibody in SSc overall, usually seen in lcSSc. Although ACA is the commonest antibody associated with PAH, there is no good evidence to suggest that ACA increases the risk of developing PAH.

A classification system by Nihtyanova et al. stratifies patients into seven main groups according to autoantibody and disease subtype [19]. Worst survival was seen in Scl70+ dcSSc patients with $41 \%$ survival at 20 years. This group had the highest incidence of ILD (91\%), and significant cardiac SSc (14\%). ACA + lcSSc patients had the highest survival (72\%), and lowest incidence of ILD (13\%). The incidence of PAH was similar to the mean for the whole cohort in this group. ARA subjects, irrespective of disease subtype, had the highest incidence of SRC (32\% at 20 years), and U3RNP+ patients the highest incidence of PAH (40\%) at 20 years.

\section{Stratification by serum biomarkers}

A useful biomarker for personalised medicine is one that changes with time and predicts disease progression or resolution. A number of different markers have been investigated, and those implicated downstream of TGF$\beta$ are often positively correlated with extent of skin sclerosis.

TGF- $\beta$ is one of the central cytokines in the pathogenesis of SSc and has been shown to be involved in fibrosis, inflammation, and vasculopathy in SSc [20]. Its activity promotes collagen synthesis, secretion, processing, and cross-linking, and it results in the secretion of key matrix molecules such as fibronectin and thrombospondin. Although it is found to be highly expressed in the tissue samples of dcSSc patients, circulating TGF- $\beta$ is not consistently elevated, and thus this makes it a less reliable biomarker.

Circulating markers correlating with skin fibrosis include interleukin-6 (IL-6) [21] and IL-13 [22]. Serum IL-6 levels have shown significant association with the extent of skin thickness, and where elevated, there is a strong associated with more severe disease at 3 years and a worse prognosis. Raised IL- 6 concentrations were also predictive of FVC and DLCO decline within the first year, and death within the first 30 months from diagnosis [23]. Elevated levels of PDGF were found to correlate with disease

Table 1. Antibodies associated with systemic sclerosis, the frequency of disease subtype within that antibody, and the frequency of organ complications for each antibody. $d c S S C$ diffuse cutaneous systemic sclerosis, LCSSc limited cutaneous systemic sclerosis, ILD interstitial lung disease, $P A H$ pulmonary arterial hypertension, $\operatorname{SRC}$ scleroderma renal crisis $[2,7,13]$

\begin{tabular}{lll}
\hline & Disease subset & Risk of organ involvement \\
Anti-centromere & $95 \%$ lcSSc & Digital ulceration \\
Anti-topoisomerase & $70 \%$ dcSSc & $70 \%$ ILD \\
Anti-RNA-Polymerase III & $>80 \%$ dcSSc & $45 \%$ SRC, malignancy, PAH \\
Anti-U3-RNP & $66 \%$ dcSSc & PAH \\
& & Cardiac \\
Anti-Th/To & LcSSc & $31 \%$ myositis \\
& & $25 \%$ PAH (all types) \\
Anti-Pm-Scl & 0verlap & $45 \%$ lung fibrosis \\
Anti-U1RNP & $80 \%$ lcssc overlap & $>50 \%$ muscle involvement \\
& & $25 \%$ ILD \\
Anti-Ku & & $25 \%$ myositis \\
Anti-U11/12 & 0verlap & 0 verlap features
\end{tabular}


activity and lung disease [24]. IL-10 overexpression correlated strongly with both skin thickening, lung involvement, and renal involvement. COMP (cartilage oligomeric matrix protein) has been shown to have close correlation with mRSS both cross-sectionally and longitudinally [25], and acts as a predictor for mortality if measured during the first 5 years of disease [26].

Connective tissue growth factor (CTGF) enhances fibroblast proliferation and acts as a downstream mediator of TGF- $\beta$ (Fig. 1). Serum CTGF correlates with extent of skin sclerosis, as well as severity of pulmonary fibrosis, as measured by DLCO and FVC [27]. It was suggested that CTGF may be more involved in maintenance of fibrosis, rather than the initiation, given levels at $<1$-year disease duration were lower than 1-3-year duration.

Elevated levels of IL-12 seem to correlate with regression of skin thickening and are found to be upregulated in affected skin of late-stage dcSSc compared to earlier disease, while MCP-1 and IL- 6 decreased with time [28], though this finding has not been upheld consistently. IL-35, a member of the IL-12 family, has also been shown to be negatively correlated with disease duration, but concentrations are not correlated with disease activity [29]. Emerging data support its role in disease initiation and collagen release resulting in increased fibrosis [29], but may not be a predictor of disease response.

MCP-1 (also known as CCL2) and MCP-3 have repeatedly been shown to have a pivotal role not only in both skin fibrosis but also in the severity of ILD [30, 31]. Higher serum concentrations of MCP-1 were predictive of a faster decline in FVC in early SSc, whereas serum IL-10 is possibly predictive of a slower decline in FVC compared to MCP-1. Improvements in mRSS correlate with a reduction in serum MCP-1 levels [32].

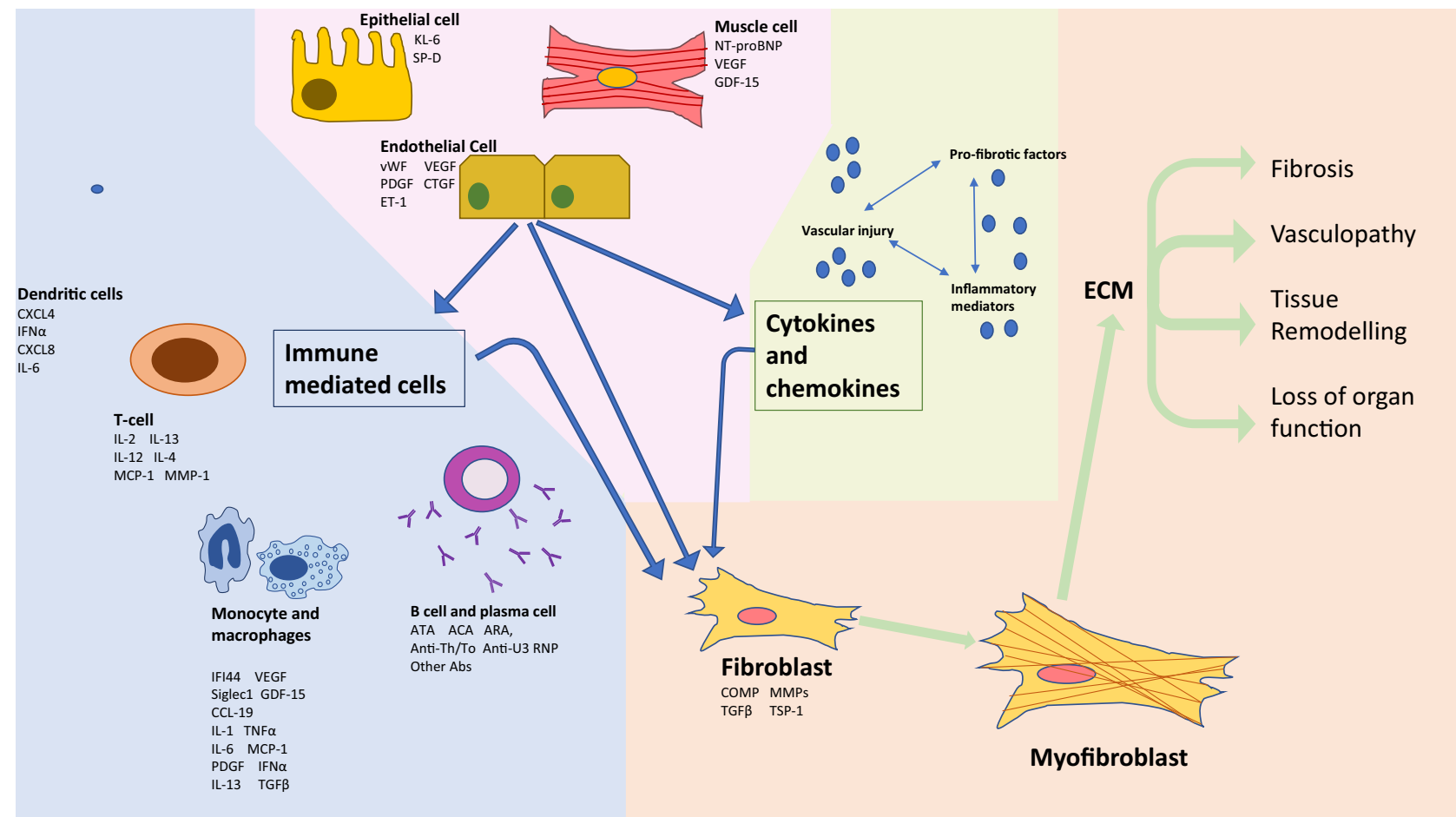

Fig. 1. Schematic highlighting the pathogenesis of SSc, with some of the potential molecular markers used for the stratification of disease in SSC, and their origin. KL-6 Krebs con den Lungen-6, SP-D surfactant protein D, vWF von Willebrand factor, PDGF platelet derived growth factor, ET-1 Endothelin-1, VEGF vascular endothelial growth factor, CTGF connective tissue growth factor, NT-proBNP $\mathrm{N}$-terminal-pro B-type natriuretic peptide, GDF-15 Growth differentiation factor 15, ECM extracellular matrix, COMP cartilage oligomeric matrix protein, TGF- $\beta$ transforming growth factor $\beta$, MMP matrix metalloproteinase, TSP-1 thrombospondin 1, ATA antitopoisomerase antibody, ACA anti-centromere antibody, ARA anti-RNA polymerase III, TNFa Tissue necrosis factor alpha, IL interleukin, MCP methyl-accepting chemotaxis protein, IFNa interferon alpha, CCL C-C Motif chemokine Ligand, CXCL C-X-C motif ligand. 
Heat shock protein 70 (HSP 70) shows positive correlation with MCP-1 and thus also modified Rodnan skin score, renal vascular resistance, and pulmonary fibrosis, and therefore may act as a supporting serological marker for fibrosis and disease severity with MCP-1 [33].

CXCL4 (or platelet factor 4) is another chemokine which has been shown to predict disease progression in SSc [34]. It is a chemotactic factor for neutrophils, fibroblasts and monocytes, and highly associated with lung and skin fibrosis, and development of pulmonary arterial hypertension.

Plasma Von Willebrand factor (vWf) is a marker of endothelial cell activation, and levels have been shown to correlate with disease severity (as measured by Medsger disease activity score), and early pulmonary involvement. They even were able to predict future development of elevated pulmonary arterial pressure in lcSSc by logistic regression models [35]. Predictors of disease onset means that high-risk individuals can be picked up prior to development of complications, and earlier diagnosis and treatment can be implicated.

These biomarkers offer the opportunity for key predictors of disease severity and have the potential to aid in predicting patient subgroups in order to modify screening for organ assessment, or target earlier interventional therapy. With time, they may even be able to be used as predictors of treatment response, but this remains to be confirmed. Individual biomarkers have not proven a strong enough association to be utilised in clinical practice yet.

\section{Stratification by genes and gene expression}

Large-scale genome-wide association studies (GWAS) have identified several candidate genes involved in SSc: consistently the major histocompatibility complex (MHC), and those involved in regulation of innate immunity, B and $\mathrm{T}$ cell activation. The first robust GWAS in SSc identified the strongest association at the 6p21 locus corresponding to the MHC. It also identified five non-human leukocyte antigen (HLA) loci: STAT4, TNPO3/IRF5, CD247, CDH7, and EXOC2/IRF4 [36]. There is significant overlap with other autoimmune diseases, specifically CD247, a mediator of T cell receptor signalling, which is implicated in systemic lupus erythematosus [37].

\section{Molecular signatures in skin}

Milano et al. [38] reported distinct gene expression profiles on skin biopsies from different subgroups of scleroderma patients. Broadly they were able to categorise the profiles into four distinct groups: fibro-proliferative, inflammatory, limited, and normal-like. The fibroproliferative group largely consisted of patients with diffuse disease, with genes associated with cell cycle and cell growth. The inflammatory signature was seen in a mixture of biopsies from diffuse, limited, and morphea patients, and was categorised by immune response and defence response genes. The limited subgroup was predominantly limited patients with a distinct gene expression profile not otherwise expressed in the other subsets, but did show high levels of variability. Lastly the normal-like group consisted mainly of healthy controls (and two SSc patients) and had increased expression of genes associated with fatty acid metabolism and lacked any expression associated with inflammation or proliferation.

Further work on dermal fibroblasts [39] confirmed that the fibroproliferative set most strongly correlated with the platelet-derived growth factor (PDGF) pathway, thus supporting the association with cell cycle and proliferation. The inflammatory subgroup showed correlation with several pathways including IL4, TNF $\alpha$, and NF B expression. Both of these two subsets correlated with TGF- $\beta$ expression, thus suggesting that TGF- $\beta$ signalling spans both subsets and is associated with more severe disease [40]. Only a weak association was seen between the inflammatory subset and interferon alpha (IFN $\alpha$ ) signalling pathway, and this was limited to the patients with early disease.

There is some evidence suggesting that these subsets may be a continuum, and patients can transition from the inflammatory subset to the normal-like state when their disease becomes inactive. Those in the normal-like therefore tend to have the longest disease duration [41].

This concept was applied by Hinchcliff et al. to treatment responders and non-responders. Those that respond to mycophenolate mofetil (MMF) predominantly expressed an inflammatory profile, whereas none of those from the fibroproliferative subset improved on MMF [42]. Identification of the intrinsic subset of patients with SSc therefore may help guide treatment choice and predict those likely to respond to treatment.

Through RNA expression analysis, Farina et al. were able to identify four key gene expression biomarkers 
which helped predict skin disease in dcSSc. These were two TGF- $\beta$ regulated genes: cartilage oligomeric matrix protein (COMP) and thrombospondin 1 (TSP-1), as well as interferon (IFN) regulated genes interferoninducible 22 (IFI44) and sialoadhesion (SIGLEC-1) [43]. These were subsequently validated, and shown to change over time with changes in mRSS. The impact of this 4-gene predictor would allow for a more objective robust measure of improvement in skin disease over mRSS for outcome in clinical trials. Longitudinal studies utilising microarray analysis later identified predominant clusters of gene correlating to the skin score, including those with profibrotic/TGF- $\beta$ regulated genes, interferon-regulated/proteasome genes, macrophage and vascular marker genes. Longitudinally THBS1 and MS4A4A correlated best with changing skin score. IFI44 was found to have a very low correlation with mRSS [44•]; thus, it is not consistently considered a good predictive marker.

Mahoney et al. looked at genetic polymorphisms identified in GWAS and the connection to intrinsic gene expression subsets from publically available data [45]. Several gene expression modules are conserved and associated with intrinsic subsets. Network analysis of these modules corresponding to the fibroproliferative and inflammatory intrinsic subsets showed five distinct and interacting subnetworks: adaptive immunity, interferon, cell proliferation M2 macrophages, and ECM. Genes linked to known SSc polymorphisms were found to interact mainly within the inflammatory subnetwork (e.g., IFR5, IRF7, IRF8, and NOTCH4), suggesting that initiating events stem from aberrant immune responses. The most important genes in SSc pathogenesis were identified as either being integral in subnetwork hubs (e.g., IFI44 for the interferon subnetwork), or connected subnetworks (e.g., PLAUR was found to connect ECM, M2 macrophage and adaptive immunity subnetworks). The strongest connections in disease subgroups were between the inflammatory and fibroproliferative intrinsic subsets, and these are linked via the ECM subnetwork and TGF- $\beta$ signalling.

Lofgren et al. identified a 415 gene signature of differentially expressed genes in skin biopsies [46]. This correlated significantly with mRSS, and any changes between this 4 S (SSc skin severity score based on difference between mean of overexpressed genes and mean of underexpressed genes in the 415 gene set) at baseline to 12 months predicted subsequent changes in skin disease at 24 months. This still requires longitudinal validation but has the potential to enable clinicians to identify early treatment response and implement any changes to therapy if no response is predicted.

\section{Targeted treatments}

Targeted therapy is already applied in other autoimmune diseases, and steps have already been made using these techniques to target therapy to the right subgroups of SSc.

Fresolimumab Fresolimumab is a human IgG4 $\mathrm{K}$ monoclonal antibody which neutralises all mammalian isoforms of TGF- $\beta$. This was trialled in patients with early dcSSc, with biomarkers of disease activity used as the primary endpoint. There was significant decline in THBS1 after 7 weeks of treatment [47]. COMP was measured as a marker of decreased skin activity, and with no significant difference compared to baseline. There was also a significant decrease in the expression levels of SERPINE1 and CTGF, both TGF- $\beta$ regulated genes. Fresolimumab seemed to have a greater impact on mRSS in patients who had higher THBS1 levels at baseline.

Some patients responded more than others, so it may be those expressing higher TGF- $\beta$ signal this targeted treatment should be applied to. Further studies are required to confirm long-term benefit from Fresolimumab.

Abatacept A recent small clinical trial utilising Abatacept, a CTLA4-Ig fusion protein, compared to placebo, decreased gene expression of inflammatory pathways in the skin following treatment that were observed in those that showed improvement in their mRSS. Two placebo patients and one treatment arm patient who did not improve over the course of the study showed stable gene expression at the two time points ( 24 weeks). The patients that did improve tended to be in the "inflammatory intrinsic subset" at baseline [48]. This suggests that there is potential to target treatment based on intrinsic subsets and therefore aid in predicting which patient subgroups are more likely to improve or not.

Tocilizumab Another example of targeted therapy came from the studies of IL-6 in SSc. Khan et al. identified a cohort of patients identifiable by diffuse skin disease, raised platelets and CRP, who had an increased IL-6 signature originating from the fibroblasts in their skin biopsies [21]. 
This led to a placebo-controlled phase II clinical trial (faSScinate). There was a near complete reversal of the proinflammatory gene expression profile on skin biopsy after 6 months with the anti-IL- 6 therapy; however, the clinical response was not so profound; with a trend towards skin improvement, and stabilisation of lung disease [49]. Given the molecular response was so profound, it raises the question that although this targeted treatment reverses the pro-inflammatory pathway in the explant fibroblasts, it most likely only stops de novo fibrosis but is unable to reverse already established fibrosis $[50 \bullet \bullet$. Therefore, a combination therapy may be required in this subgroup of patients. At time of writing, the phase III trial is yet to report.

Dasatinib Dasatinib is a tyrosine kinase inhibitor with anti-fibrotic efficacy [51], as well as inhibiting $\mathrm{T}$ cell receptor signalling and the production of inflammatory and fibrotic cytokines [52]. Gene expression data from skin biopsies of patients treated with dasatinib for ILD were analysed. Of those that improved (defined as change in mRSS $>5$ points or decrease $>20 \%$ from baseline), their gene expression subset was consistent with fibroproliferative or normal-like subsets, whereas the majority of non-improvers were in the inflammatory subset [53]. The pathways that were downregulated post-treatment in improvers included fibrotic gene subsets (e.g., PI3K/AKT/MTOR signalling and TGF- $\beta$ signalling), as well as inflammatory pathways including IFN $\alpha /$ IFN $\gamma$ response and TNF $\alpha / N F$ B signalling, whereas in the non-improvers, the gene expression in these pathways did not change.
A recent meta-analysis of clinical trials in SSc compared the gene expression patterns in skin samples of patients treated with mycophenolate mofetil, abatacept, rituximab, fresolimumab, and nilotinib. It found that regardless of therapy used, underpinning most clinical improvements was a resolution of inflammatory pathways found in the gene expression at baseline [54•]. Treatment response was more commonly associated with higher activity of ECM related genes, highlighting its central role in SSc network activity. Biological differences were noted in the patients in the different therapy subgroups and thus have the potential of being integrated to guide treatment choice. This was particularly highlighted in the patients who did not respond to fresolimumab and had high baseline expression of inflammatory pathways that were well targeted with MMF.

Franks et al. utilised their intrinsic subset and baseline and 48/54 week serum samples to gain insight into those who are more likely to respond to haematopoietic stem cell transplant (HSCT) [55]. They found that participants in the normal-like subset did not gain clinical improvement of receiving HSCT over cyclophosphamide. However, those in the fibroproliferative subset did receive the most benefit from HSCT, and this subgroup tends to be the group they have previously reported as non-responders to standard therapy (MMF or abatacept).

These clinical findings therefore suggest that patients that fall in the inflammatory subset should be treated with MMF or abatacept, while those in the fibroproliferative group are more likely to respond to HSCT, dasatinib and fresolimumab, as are the normallike (but not HSCT).

\section{Conclusion}

Recent advances in high throughput analysis technologies (transcriptomics and proteomics) has not only broadened our understanding of disease but allowed us to have a genome-wide view of molecular variation in disease and healthy state. They also allow for a molecular understanding of changes in response to treatment and variations in heterogenous disease.

Stratified and personal medicine relies on these high output platforms providing multiplex analysis data and next-generation sequencing platforms in order to understand how disease states respond to therapies, what the changes are in those that benefit from treatment compared to those that do not, and ultimately help in ensuring that the correct patient 
is identified who will gain benefit both at a molecular and clinical level from these interventions, without subjecting the risks of any medication with no perceived benefit.

Scleroderma is a heterogenous disease, and thus far no one biomarker, gene, or gene expression data can fully explain the variability in both trajectory and response to therapy. Gene expression profiling offers larger scale molecular understanding of the disease process at different timepoints, with the ability to identify patient subsets by utilising these resources. These subsets clearly respond differently to treatments as well, and clearer stratification will identify those most likely to respond to the disease, early signatures of treatment response, and ultimately increase the power of a successful clinical trial. This is all vital in order to identify the right treatment for the right patient at the right time. This vision of stratified and personalised medicine is becoming increasingly achievable and has already been applied in the use of IL- 6 therapy. Stratifying patients into those who are more likely to respond to certain therapies will make a dramatic difference in clinical trials and ensure earlier access to appropriate therapies in the near future.

\section{Compliance with Ethics Guidelines}

\section{Conflict of Interest}

The authors declare that they have no conflict of interest.

\section{Human and Animal Rights and Informed Consent}

This article does not contain any studies with human or animal subjects performed by any of the authors.

Open Access This article is distributed under the terms of the Creative Commons Attribution 4.0 International License (http://creativecommons.org/licenses/by/4.0/), which permits unrestricted use, distribution, and reproduction in any medium, provided you give appropriate credit to the original author(s) and the source, provide a link to the Creative Commons license, and indicate if changes were made.

\section{References and Recommended Reading}

Papers of particular interest, published recently, have been highlighted as:

- Of importance

$\bullet \quad$ Of major importance

1. Deng X, Nakamura Y. Cancer precision medicine: from Cancer screening to drug selection and personalized immunotherapy. Trends Pharmacol Sci. 2017;38(1):15-24.

2. Nihtyanova SI, Denton CP. Autoantibodies as predictive tools in systemic sclerosis. Nat Rev Rheumatol. 2010;6(2):112-6.

3. Biomarkers Definitions Working Group. Biomarkers and surrogate endpoints: preferred definitions and conceptual framework. Clin Pharmacol Ther. 2001;69(3):89-95.

4. Khanna D, Furst DE, Clements PJ, Allanore Y, Baron M, Czirjak L, et al. Standardization of the modified Rodnan skin score for use in clinical trials of systemic sclerosis. J Scleroderma Relat Disord. 2017;2(1):11-8. 5. Shand L, Lunt M, Nihtyanova S, Hoseini M, Silman A, Black CM, et al. Relationship between change in skin score and disease outcome in diffuse cutaneous 
systemic sclerosis: application of a latent linear trajectory model. Arthritis Rheum. 2007;56(7):2422-31.

6. Herrick AL, Pan X, Peytrignet S, Lunt M, Hesselstrand R, Mouthon L, et al. Treatment outcome in early diffuse cutaneous systemic sclerosis: the European scleroderma observational study (ESOS). Ann Rheum Dis. 2017;76(7):1207-18.

7. Nihtyanova SI, Schreiber BE, Ong VH, Rosenberg D, Moinzadeh P, Coghlan JG, et al. Prediction of pulmonary complications and long-term survival in systemic sclerosis. Arthritis Rheum (Hoboken, NJ).

2014;66(6):1625-35.

8. Wu W, Jordan S, Becker MO, Dobrota R, Maurer B, Fretheim H, et al. Prediction of progression of interstitial lung disease in patients with systemic sclerosis: the SPAR model. Ann Rheum Dis. 2018;77(9):1326-32.

9. Elhai M, Hoffmann-Vold AM, Avouac J, Pezet S, Cauvet A, Leblond A, et al. Performance of candidate serum biomarkers for systemic sclerosis-interstitial lung disease. Arthritis Rheum (Hoboken, NJ). 2019. https:// doi.org/10.1002/art.40815.

10. Goh NS, Desai SR, Veeraraghavan S, Hansell DM, Copley SJ, Maher TM, et al. Interstitial lung disease in systemic sclerosis: a simple staging system. Am J Respir Crit Care Med. 2008;177(11):1248-54.

11. Maher TM, Oballa E, Simpson JK, Porte J, Habgood A, Fahy WA, et al. An epithelial biomarker signature for idiopathic pulmonary fibrosis: an analysis from the multicentre PROFILE cohort study. Lancet Respir Med. 2017;5(12):946-55.

12. Jenkins RG, Simpson JK, Saini G, Bentley JH, Russell AM, Braybrooke R, et al. Longitudinal change in collagen degradation biomarkers in idiopathic pulmonary fibrosis: an analysis from the prospective, multicentre PROFILE study. Lancet Respir Med. 2015;3(6):462-72.

13. Kolstad KD, Li S, Steen V, Chung L. Long-term outcomes in systemic sclerosis-associated pulmonary arterial hypertension from the pulmonary hypertension assessment and recognition of outcomes in scleroderma registry (PHAROS). Chest. 2018;154(4):862-71.

14. Allanore Y, Borderie D, Avouac J, Zerkak D, Meune C, Hachulla E, et al. High N-terminal pro-brain natriuretic peptide levels and low diffusing capacity for carbon monoxide as independent predictors of the occurrence of precapillary pulmonary arterial hypertension in patients with systemic sclerosis. Arthritis Rheum.

2008;58(1):284-91.

15. Coghlan JG, Denton CP, Grunig E, Bonderman D, Distler O, Khanna D, et al. Evidence-based detection of pulmonary arterial hypertension in systemic sclerosis: the DETECT study. Ann Rheum Dis. $2014 ; 73(7): 1340-9$.

16. Papaioannou AI, Zakynthinos E, Kostikas K, Kiropoulos T, Koutsokera A, Ziogas A, et al. Serum VEGF levels are related to the presence of pulmonary arterial hypertension in systemic sclerosis. BMC Pulm Med. 2009;9:18.
17. Meadows CA, Risbano MG, Zhang L, Geraci MW, Tuder RM, Collier DH, et al. Increased expression of growth differentiation factor-15 in systemic sclerosisassociated pulmonary arterial hypertension. Chest. 2011;139(5):994-1002.

18. Stratton RJ, Pompon L, Coghlan JG, Pearson JD, Black $\mathrm{CM}$. Soluble thrombomodulin concentration is raised in scleroderma associated pulmonary hypertension. Ann Rheum Dis. 2000;59(2):132-4.

19. Nihtyanova SID-SE, Fonseca CM, Ong VH, Denton CP. A practical classification of systemic sclerosis using subset and autoantibodies for the purpose of early risk stratification. Arthritis Rheum (Hoboken, NJ). 2018;70(suppl 10):2935.

20. Lafyatis R. Transforming growth factor beta-at the Centre of systemic sclerosis. Nat Rev Rheumatol. 2014;10(12):706-19.

21. Khan K, Xu S, Nihtyanova S, Derrett-Smith E, Abraham $\mathrm{D}$, Denton CP, et al. Clinical and pathological significance of interleukin 6 overexpression in systemic sclerosis. Ann Rheum Dis. 2012;71(7):1235-42.

22. Fuschiotti P, Medsger TA Jr, Morel PA. Effector CD8+ T cells in systemic sclerosis patients produce abnormally high levels of interleukin-13 associated with increased skin fibrosis. Arthritis Rheum. 2009;60(4):1119-28.

23. De Lauretis A, Sestini P, Pantelidis P, Hoyles R, Hansell DM, Goh NS, et al. Serum interleukin 6 is predictive of early functional decline and mortality in interstitial lung disease associated with systemic sclerosis. J Rheumatol. 2013;40(4):435-46.

24. Trojanowska M. Role of PDGF in fibrotic diseases and systemic sclerosis. Rheumatology (Oxford, England). 2008;47(Suppl 5):v2-4.

25. Hesselstrand R, Kassner A, Heinegard D, Saxne T. COMP: a candidate molecule in the pathogenesis of systemic sclerosis with a potential as a disease marker. Ann Rheum Dis. 2008;67(9):1242-8.

26. Hesselstrand R, Andreasson K, Wuttge DM, Bozovic G, Scheja A, Saxne T. Increased serum COMP predicts mortality in SSc: results from a longitudinal study of interstitial lung disease. Rheumatology (Oxford, England). 2012;51(5):915-20.

27. Sato $S$, Nagaoka T, Hasegawa M, Tamatani $T$, Nakanishi T, Takigawa M, et al. Serum levels of connective tissue growth factor are elevated in patients with systemic sclerosis: association with extent of skin sclerosis and severity of pulmonary fibrosis. J Rheumatol. 2000;27(1):149-54.

28. Matsushita T, Hasegawa M, Hamaguchi Y, Takehara K, Sato S. Longitudinal analysis of serum cytokine concentrations in systemic sclerosis: association of interleukin 12 elevation with spontaneous regression of skin sclerosis. J Rheumatol. 2006;33(2):275-84.

29. Tomcik M, Zerr P, Palumbo-Zerr K, Storkanova H, Hulejova H, Spiritovic M, et al. Interleukin-35 is upregulated in systemic sclerosis and its serum levels are 
associated with early disease. Rheumatology (Oxford, England). 2015;54(12):2273-82.

30. Wu M, Baron M, Pedroza C, Salazar GA, Ying J, Charles $\mathrm{J}$, et al. CCL2 in the circulation predicts long-term progression of interstitial lung disease in patients with early systemic sclerosis: data from two independent cohorts. Arthritis Rheum (Hoboken, NJ).

2017;69(9):1871-8.

31. Yanaba K, Komura K, Kodera M, Matsushita T, Hasegawa M, Takehara K, et al. Serum levels of monocyte chemotactic protein-3/CCL7 are raised in patients with systemic sclerosis: association with extent of skin sclerosis and severity of pulmonary fibrosis. Ann Rheum Dis. 2006;65(1):124-6.

32. Hasegawa M, Fujimoto $M$, Matsushita T, Hamaguchi $Y$, Takehara K, Sato S. Serum chemokine and cytokine levels as indicators of disease activity in patients with systemic sclerosis. Clin Rheumatol. 2011;30(2):231-7.

33. Ogawa F, Shimizu K, Hara T, Muroi E, Hasegawa M, Takehara K, et al. Serum levels of heat shock protein 70, a biomarker of cellular stress, are elevated in patients with systemic sclerosis: association with fibrosis and vascular damage. Clin Exp Rheumatol.

2008;26(4):659-62.

34. van Bon L, Affandi AJ, Broen J, Christmann RB, Marijnissen RJ, Stawski L, et al. Proteome-wide analysis and CXCL4 as a biomarker in systemic sclerosis. N Engl J Med. 2014;370(5):433-43.

35. Barnes T, Gliddon A, Dore CJ, Maddison P, Moots RJ. Baseline vWF factor predicts the development of elevated pulmonary artery pressure in systemic sclerosis. Rheumatology (Oxford, England). 2012;51(9):1606-9.

36. Radstake TR, Gorlova O, Rueda B, Martin JE, Alizadeh BZ, Palomino-Morales R, et al. Genome-wide association study of systemic sclerosis identifies CD247 as a new susceptibility locus. Nat Genet. 2010;42(5):426-9.

37. Gorman CL, Russell AI, Zhang Z, Cunninghame Graham D, Cope AP, Vyse TJ. Polymorphisms in the CD3Z gene influence TCRzeta expression in systemic lupus erythematosus patients and healthy controls. J Immunol (Baltimore, Md : 1950). 2008;180(2):1060-70.

38. Milano A, Pendergrass SA, Sargent JL, George LK, McCalmont TH, Connolly MK, et al. Molecular subsets in the gene expression signatures of scleroderma skin. PLoS One. 2008;3(7):e2696.

39. Johnson ME, Mahoney JM, Taroni J, Sargent JL, Marmarelis E, Wu MR, et al. Experimentally-derived fibroblast gene signatures identify molecular pathways associated with distinct subsets of systemic sclerosis patients in three independent cohorts. PLoS One. 2015;10(1):e0114017.

40. Sargent JL, Milano A, Bhattacharyya S, Varga J, Connolly MK, Chang HY, et al. A TGFbeta-responsive gene signature is associated with a subset of diffuse scleroderma with increased disease severity. J Invest Dermatol. 2010;130(3):694-705.
41. Assassi S, Swindell WR, Wu M, Tan FD, Khanna D, Furst DE, et al. Dissecting the heterogeneity of skin gene expression patterns in systemic sclerosis. Arthritis Rheum (Hoboken, NJ). 2015;67(11):3016-26.

42. Hinchcliff M, Huang CC, Wood TA, Matthew Mahoney J, Martyanov V, Bhattacharyya S, et al. Molecular signatures in skin associated with clinical improvement during mycophenolate treatment in systemic sclerosis. J Investig Dermatol. 2013;133(8):1979-89.

43. Farina G, Lafyatis D, Lemaire R, Lafyatis R. A four-gene biomarker predicts skin disease in patients with diffuse cutaneous systemic sclerosis. Arthritis Rheum. 2010;62(2):580-8.

44. $\quad$ Rice LM, Ziemek J, Stratton EA, McLaughlin SR, Padilla $\mathrm{CM}$, Mathes AL, et al. A longitudinal biomarker for the extent of skin disease in patients with diffuse cutaneous systemic sclerosis. Arthritis Rheum (Hoboken, NJ). 2015;67(11):3004-1.

Utilising gene expression data to predict skin score, thus highlighting key biomarkers corresponding to skin progression in SSc.

45. Mahoney JM, Taroni J, Martyanov V, Wood TA, Greene CS, Pioli PA, et al. Systems level analysis of systemic sclerosis shows a network of immune and profibrotic pathways connected with genetic polymorphisms. PLoS Comput Biol. 2015;11(1):e1004005.

46. Lofgren S, Hinchcliff M, Carns M, Wood T, Aren K, Arroyo E, et al. Integrated, multicohort analysis of systemic sclerosis identifies robust transcriptional signature of disease severity. JCI Insight. 2016;1(21):e89073.

47. Rice LM, Padilla CM, McLaughlin SR, Mathes A, Ziemek J, Goummih S, et al. Fresolimumab treatment decreases biomarkers and improves clinical symptoms in systemic sclerosis patients. J Clin Invest. 2015;125(7):2795-807.

48. Chakravarty EF, Martyanov V, Fiorentino D, Wood TA, Haddon DJ, Jarrell JA, et al. Gene expression changes reflect clinical response in a placebo-controlled randomized trial of abatacept in patients with diffuse cutaneous systemic sclerosis. Arthritis Res Ther. 2015;17:159.

49. Khanna D, Denton CP, Jahreis A, van Laar JM, Frech TM, Anderson ME, et al. Safety and efficacy of subcutaneous tocilizumab in adults with systemic sclerosis (faSScinate): a phase 2, randomised, controlled trial. Lancet (London, England). 2016;387(10038):2630-40.

$50 . \bullet$ Denton CP, Ong VH, Xu S, Chen-Harris H, Modrusan $\mathrm{Z}$, Lafyatis $\mathrm{R}$, et al. Therapeutic interleukin-6 blockade reverses transforming growth factor-beta pathway activation in dermal fibroblasts: insights from the faSScinate clinical trial in systemic sclerosis. Ann Rheum Dis. 2018;77(9):1362-71

Clear insights into the exact effect IL-6 blockade has on the fibroblasts in SSc through gene expression and pathway analysis.

51. Akhmetshina A, Dees C, Pileckyte M, Maurer B, Axmann R, Jungel A, et al. Dual inhibition of c-abl and 
PDGF receptor signaling by dasatinib and nilotinib for the treatment of dermal fibrosis. FASEB J. 2008;22(7):2214-22.

52. Schade AE, Schieven GL, Townsend R, Jankowska AM, Susulic V, Zhang R, et al. Dasatinib, a small-molecule protein tyrosine kinase inhibitor, inhibits T-cell activation and proliferation. Blood. 2008;111(3):1366-77.

53. Martyanov V, Kim GJ, Hayes W, Du S, Ganguly BJ, Sy $\mathrm{O}$, et al. Novel lung imaging biomarkers and skin gene expression subsetting in dasatinib treatment of systemic sclerosis-associated interstitial lung disease. PLoS One. 2017;12(11):e0187580.

54. Taroni JN, Martyanov V, Mahoney JM, Whitfield ML. A functional genomic meta-analysis of clinical trials in systemic sclerosis: toward precision medicine and combination therapy. J Investig Dermatol. 2017;137(5):1033-41

Meta-analysis looking a groups of patients treated with different therapies, and lessons we can learn in selecting the correct patients for targeted treatment.
55. Franks JMV, Wood TA, Crofford L, Keyes-Elstein L, Furst DE, Goldmuntz E, et al. Machine learning classification of peripheral blood gene expression identifies a subset of patients with systemic sclerosis Most likely to show clinical improvement in response to hematopoietic stem cell transplant [abstract]. Arthritis Rheumatol (Hoboken, NJ). 2018;70(suppl 10).

\section{Publisher's Note}

Springer Nature remains neutral with regard to jurisdictional claims in published maps and institutional affiliations. 